\title{
Bases para la Atención Ginecológica Especializada a Niñas y Adolescentes
}

\author{
Dr. Alberto Duarte-Contreras, M.M.*
}

Cada día se hace más palpable la necesidad de brindar a los médicos la oportunidad del estudio y del entrenamiento en los problemas ginecológicos de las niñas y gineco-obstétricos de las adolescentes.

Hace cinco décadas eran prácticamente desconocidos los cursos, los libros y los trabajos que se ocupaban de estas disciplinas. En 1939 el pediatra húngaro L. Dobszay publica el primer libro que se conoce sobre Ginecología Pediátrica. En 1940 el ginecólogo checoeslovaco R. Peter organiza en Praga un servicio hospitalario de Ginecología para Niñas y Adolescentes y en 1953 la Universidad de Charles, de Praga, funda para él la Cátedra de Ginecología Pediátrica: son el primer Servicio y la primera Cátedra Universitaria de Ginecología InfantoJuvenil de que se tenga noticia en la historia de la medicina. Desde entonces, con la preocupación imperante en todas las esferas por la profilaxis y pronta solución de los problemas de salud, las alteraciones morfológicas y funcionales del aparato genital de la niña y de la adolescente han despertado la atención de los padres y el interés de las Instituciones $y$ del cuerpo médico en general.

Por ello vemos que el estudio de los problemas ginecológicos que podemos

\footnotetext{
Presidente de la Sociedad Colombiana de Ginecología Juvenil y Pediátrica. Miembro de Número de la Academia Nacional de Medicina de Colombia.
}

encontrar en los primeros años de la vida forma parte de los programas de educación de un buen número de Facultades de Medicina y que muchos Departamentos Gíneco-Obstétricos de los grandes hospitales, cuentan con Servicios de Con sulta Externa y salones destinados exclusivamente para la atención de este grupo de pacientes, a más del sin número de cursos que se adelantan a todo nivel para la preparación del personal médico $y$ paramédico.

Si algún campo es complejo para su estudio a esta edad, es el de la Ginecología, campo que se debe analizar desde la vida embrionaria; por ello los trastornos ginecológicos durante la niñez y la adolescencia han ganado la atención creciente del progreso médico.

El tratamiento y el comportamiento médico ante estas pequeñas pacientes jamás podrá limitarse a la solución de las alteraciones fisiológicas o morfológicas: nos llevará siempre a profundizar en sus problemas sicológicos, con un análisis diferente al requerido por la mujer adulta, puesto que no tratamos problemas o trastornos, sino personas, que llegarán o están pasando rápida y bruscamente a la mayor edad. Ello explica nuestro interés por la orientación moderna que estamos dando a nuestra especialidad, la más antigua de la humanidad.

Los problemas emocionales, sociales y morales que lleva consigo la maternidad 
a tan temprana edad, máxime cuando se trata de solteras y niñas sin apoyo, más los peligros de contagio y trasmisión de enfermedades venereas, producto de la promiscuidad, hacen aún más difícil el trabajo del médico tratante.

No es para nosotros un secreto la ignorancia familiar en lo que atañe a las alteraciones ginecológicas a esta edad, ni el encierro engañoso en el que se quiere ocultar y manejar un embarazo en los primeros años del despertar juvenil.

Conocemos las limitaciones económicas de la mayoría de los Hospitales de todos los países, y por ello somos concientes del porqué, salvo algunas excepciones, no cuentan con servicios exclusivos para la atención ginecológica de estas pacientes. Reconocemos también la falta de preparación adecuada del cuerpo médico para afrontar con tino y responsabilidad el enfoque terapéutico y el saneamiento sicológico de infinidad de problemas que de no ser tratados, serán fuente de taras y coadyuvantes en la desmoralización de las costumbres.

Con base en estas tres premisas, podremos estudiar los métodos de atención a la niña y a la adolescente, como también la necesidad y la manera de organizar policl ínicas especializadas.

\section{Introducción a la comunidad}

Hemos tenido la oportunidad de exponer el tema "El porqué de la Ginecología Infanto Juvenil" en muchas reuniones $y$ ante diferentes estratos sociales e intelectuales; en todas hemos observado falta de conocimientos al respecto.

Nuestra primera inquietud ha de ser la de instruir a la comunidad, formándole conciencia de que toda niña desde el nacimiento puede presentar, como mujer que es, trastornos ginecológicos, anató- micos o funcionales, leves o severos que en la adolescencia está expuesta a una maternidad $o$ a una vida sexual activa, mal dirigida, con todos sus peligros, y que es deber de las personas que las tienen bajo su responsabilidad, conducirlas oportunamente a la consulta ginecológica de centros asistenciales o a consultas privadas.

Esta instrucción la podremos adelantar con facilidad y eficacia en la consulta prenatal, en las reuniones de padres de familia que organizan los centros educativos y las agrupaciones gremiales y sindicales. Todos los grandes rotativos publican la "Página Femenina": es tal vez el mejor conducto para adelantar esta educación dado el alto y ávido número de lectores. La radio y la televisión son otros excelentes medios para llevar al pueblo conocimientos oportunos.

Es indispensable informar por medio de cursos especializados a los educadores, a todo nivel; una vez motivados, nos ayudarán a formar conciencia en los educandos sobre la necesidad de la consulta ginecológica a esta edad.

Sólo cuando hayamos instruido a todas las esferas sociales sobre la importancia de la atención ginecológica a la niña a partir del nacimiento, podremos decir que se ha avanzado en lo que hoy es un proyecto en etapa inicial de desarrollo. Sólo entonces tendremos un volumen suficiente de pacientes para adelantar estudios e investigaciones; sólo entonces podremos presentar estadísticas significativas que respalden nuestros requerimientos ante las autoridades asistenciales; sólo entonces podremos iniciar con experiencia y pleno apoyo campañas de prevención de embarazos a muy temprana edad, de enfermedades venéreas, de prostitución $\mathrm{y}$ de un sinnúmero de alteraciones que afectan el futuro emocional, sexual, obstétrico y familiar de estas nuestras pequeñas pacientes. 
Si por apatía o por falta de motivación no adelantamos campañas educativas, seguirán llegando a la consulta solamente las lesiones traumáticas, las masas tumorales ya en sus últimos estadios, el abdomen agudo ginecológico, el aborto séptico, los embarazos y los partos complicados, las secuelas de las enfermedades venéreas, como sucede en la actualidad

Las Sociedades de Ginecología Infanto Juvenil son las llamadas a adelantar estas campañas. Por ello es indispensable su organización en todos los países y ellas deben ser las responsables de la atención ginecológica de niñas y adolescentes.

Cada día estamos más convencidos de que la instrucción a la comunidad es la base primordial para el éxito de nuestro cometido, y la base de los métodos de atención a esta edad.

\section{Educación médica}

El vasto carnpo y la complejidad de la ginecología en niñas y adolescentes abarca aspectos médicos, quirúrgicos, genéticos, endocrinológicos, síquicos, sociales, legales y pediátricos, que debe conocer quien se dedique a esta especialidad.

Las anomalías congénitas en el aparato genital de la recién nacida pueden convertirse en un intrincado problema pará su diagnóstico no sólo a esta edad sino también durante la pubertad, la adolescencia y los primeros años de la edad adulta. Si a esto se agregan los problemas de los estados intersexuados, las decisiones tomadas adquieren una tremenda responsabilidad. Con frecuencia estas lesiones se asocian con disgenesias gonadales, situación por demás de difícil solución. Las vulvovaginitis de toda etiología, constituyen un al to porcentaje de consulta $(1-6)$.
Los tumores del aparato genital, no tan raros como se les pretende presentar (7-15), no siempre tienen constitución ni evolución benigna. Las alteraciones endocrinas en esta etapa de perfeccionamiento somático, funcional y síquico, exigen adiestramiento especial $(2-5)$. Las lesiones no tan infrecuentes de los senos y los sangrados genitales de las niñas, de las impúberes y de las que ya se han iniciado en pubertad, constituyen, con frecuencia, un problema de solución urgente, de difícil manejo, con compromiso emocional, que requiere tino y experiencia para su solución. Los traumatismos y la agresión sexual presentan serias dificultades no sólo quirúrgicas sino también legales con un casi indeleble compromiso síquico. El incremento de la libertad sexual que coloca a las adolescentes en peligro de las enfermedades venéreas, de la promiscuidad, de la prostitución y de los embarazos no deseados a tan temprana edad, siempre de alto riesgo (8-9), conforman un capítulo que exige idoneidad para su acertada solución. La falta de planificación familiar que conduce al aborto provocado hace, con todo el cortejo sintomático y la complejidad sicológica de los puntos rsbozados, que esta especialidad se agigante y se dificulte por tan extenso panorama que ella abarca.

Preguntamos: ¿tenemos los ginecológos y tienen los pediatras la suficiente información para la solución de todos estos problemas?

El ginecólogo no está familiarizado con el trato a estas niñas, $v$ por ello con frecuencia, su presencia le coloca en situaciones embarazosas. El médico general y los pediatras, rara vez incluyen en sus exámenes de rutina el de los órganos genitales infantiles y pasan por alto, muchas veces, inquietudes formuladas por las madres.

Al revisar el informe de la F.I.G.I.J. (Federación Internacional de Ginecolo- 
gía Infanto Juvenil) presentado al $\mathrm{V}$ Simposio Internacional reunido en Tokio el 22 de octubre de 1979 (4), vemos que de 40 países que cuentan con grupos de estudio o con Sociedades de Ginecología Infanto Juvenil o con destacados profesionales dedicados a esta especialidad, solamente nueve tenían programas de docencia en la cátedra universitaria (cuadro No. 1).

Cuadro No. 1

METODOS DE ATENCION DE LA NIÑA $Y$ DE LA ADOLESCENTE

Cátedra Universitaria en 1979

\begin{tabular}{|lll|}
\hline \multicolumn{1}{|c}{ PAIS } & UNIVERSIDAD & DOCENCIA A : \\
\hline Austria & Insbruck & $\begin{array}{l}\text { Ginecólogos } \\
\text { Y Pediatras }\end{array}$ \\
Brasil & Rio de Janeiro & Estudiantes \\
Checoeslovaquia & En todas & Estudiantes \\
Finlandia & Helsinki-Tork & Estudiantes \\
Hungria & En todas & Estudiantes \\
& & Especialidad \\
Rusia & En todas & Estudiantes \\
& & Especialidad \\
Suiza & Nuniversidades & Estudiantes \\
Yugoeslavia & Estudiantes
\end{tabular}

Hoy el panorama ha cambiado $y$ observamos un asombroso progreso. Sabemos que en todos Ios países con Sociedades afiliadas a F.I.G.I.J. se están desarrollando programas universitarios para alumnos de ginecología y de pediatría, a más de programas de postgrado para formación de especialistas y de adiestramiento para médicos generales y para personal auxiliar.

Además, las Sociedades de Ginecología Infanto Juvenil organizan con frecuencia cursos y simposios nacionales e internacionales de formación y adiestramiento.
Esta inquietud, por el conocimiento de los intrincados problemas ginecológicos a esta edad, es alentadora y promisoria. Fruto de ello la extensa y bien documentada bibliografía que a diario encontramos en revistas de ginecología y de pediatría, como también en textos de estudio y de consulta.

Estamos convencidos de que en breve tendremos un selecto grupo de especialistas idóneos para la atención y solución de cuantos problemas se les puedan presentar a estas pequeñas pacientes.

En lo que concierne a Colombia, el 4 de marzo de 1971 fundamos en la ciudad de Cúcuta la Sociedad Colombiana de Ginecología Infantil y Pediátrica: fue la primera en América Latina (3). En este mismo año se constituyeron filiales en Barranquilla y Bucaramanga y se organizaron comisiones y centros de estudio en algunas sociedades locales de gínecoobstetricia. En diciembre del mismo año, con ocasión del IX Congreso Colombiano de Obstetricia y Ginecología reunido en la ciudad de Bucaramanga, se realizó bajo la presidencia honoraria de los Profesores Robert Contamin y Hubert De Wateville, la primera sesión de trabajo sobre el tema "La Ginecología en la niña y la adolescente".

Desde entonces, en nuestra condición de Vice Presidente Delegado para la América Latina (3), empezamos los contactos pertinentes con prestigiosos ginecologos de todos los países del continente americano con miras a la formación de grupos de estudio $y$ de sociedades organizadas. Lo que para nosotros en esa época fuese una ilusión, es hoy una realidad.

En 1973 la Academia Nacional de Medicina de Colombia celebró en Bogotá sus cien años de fundación con la realización de un simposio sobre "Problemas 
de la Adolescencia en Colombia" y en él se estudió ampliamente el problema ginecológico y obstétrico como también el de programas de estudio en la cátedra universitaria, a más de la organización de la Consulta Externa y de salas de hospitalización especiales para adolescentes.

En 1975 la Federación Colombiana de Sociedades de Obstetricia y Ginecología, FECOLSOG, realizó un simposio sobre "Ginecología de la niña y la adoleste", para Ginecólogos y Pediatras.

En todos los congresos nacionales de la especialidad a partir de 1971, se han dictado cursillos de actualización y se han verificado sesiones especiales para el estudio de estos tópicos.

En la Revista Colombiana de Obstetricia y Ginecología se publican al respecto, con frecuencia, trabajos de gran interés y actualidad.

En los programas de docencia de la mayoría de las facultades de medicina se ha incluido el estudio de este nuevo enfoque de nuestra especialidad.

Este panorama esbozado nos indica que el cuerpo médico de Colombia, conciente de que se necesita la formación en este campo, ha volcado todo su empeño para su preparación y la de futuras promociones en torno a una rama de la especialidad que había pasado inadvertida y que hoy se estudia con dedicación y entusiasmo.

\section{Atención hospitalaria}

La consulta externa es la base del funcionamiento de un hospital y de ella depende en gran parte el éxito o el fracaso de los servicios internos.

En el informe ya citado de la F.I.G.I.J. (3), vemos que en los grandes hospita- les de los cuarenta países a ella vinculados, existe la consulta externa especializada para niñas y adolescentes, con atención de una a cuatro horas diarias en unos y con menos horas en otros, según el requerimiento.

¿Cómo funcionan estas consultas? Veámoslo, tomando como ejemplo la de la Clínica Infantil de Cúcuta.

La niña menor de 11 años va a la consulta externa de la Clínica Infantil Teresa Briceño de Andressen, por cualquier problema general o por manifestaciones patológicas de su área genital. EI pediatra, en el caso específico, la remite al consultorio de Ginecología Pediátrica, atendido por un ginecólogo experimentado. Hace unos años, pocas eran las pacientes que asistían a esta consulta, así las hubiesen remitido los pediatras: faltaba instrucción a las usuarias y a sus allegados. Con el correr del tiempo y con la colaboración de todas las consultas pediátricas de la ciudad, ha aumentado el requerimiento $y$ se ha logrado que muchas niñas sean llevadas en solicitud directa del Servicio de Ginecología Pediátrica.

Este consultorio funciona 2 horas diarias y cuenta para su atención con la colaboración de los pediatras de la Institución. Se ha logrado de esta manera incrementar los conocimientos ginecológicos infantiles de los pediatras, y adiestrar a los ginecólogos en el manejo de las niñas.

Cuando la paciente requiere hospitalización, pasa, dentro de la misma Clínica Infantil, al Servicio de Cirugía o de Medicina Interna. Las urgencia traumáticas van directamente de emergencia a las salas de traumatología o de cirugía, según la lesión. Las pacientes hospitalizadas son tratadas por el ginecólogo en colaboración con los pediatras. Las pacientes quirúrgicas son intervenidas por 
el ginecólogo en asocio del cirujano pediatra.

Es éste el proceso seguido por una niña hasta de 11 años; a partir de esta edad se inicia el calvario para estas jovencitas. pues ya no se les atiende en la Clínica Infantil, sean impúberes o púberes. Deben entonces, acudir a los Servicios de Consulta Externa Ginecológica o al Prenatal que para personas adultas tiene el Hospital de San Juan de Dios.

Los Puestos de Salud que existen en los barrios periféricos de la ciudad remiten estas pequeñas pacientes ginecológicas, según su edad, a la Clínica Infantil $o$ al hospital general.

El primer escollo que debe afrontar esta niña mayor de 11 años, en su desorientación y la de su acompañante al verse rodeadas por pacientes de mayor edad, quienes en la mayoría de los casos se encuentran comentando sus dolencias $y$ en no pocas oportunidades, sus problemas personales y privados, no muy edificantes muchas veces.

Para la consulta no hay día fijo ni horario especial, ni prelación alguno como lo indica la simple razón. Al pasar a la consulta, el ginecólogo casi 'nunca cuenta con el tiempo suficiente disponible requerido para la atención de estas pacientes y sin la adecuada experiencia para su manejo: ello hace que sea muy deficiente el interrogatorio y que, se pase rápidamente al examen, si acaso es posible, sin ganarse la confianza de la niña que siempre va angustiada y muchas veces está asediada de prejuicios. Con inusitada frecuencia la persona acompañante, cualquiera que ella sea, poco colabora en el suministro de datos requeridos. Así, ante una niña intranquila, reacia y timorata, es lógico que la consulta sea deficiente.

Si esta jovencita requiere hospitalización, se remite al Servicio de Ginecolo- gía, donde no va a tener privacidad. La intervención quirúrgica, si es el caso, está a cargo del ginecólogo, lo mismo que todos los tratamientos requeridos.

Las adolescentes que más nos han preocupado son las embarazadas, por múltiples motivos expuestos ya por nosotros en trabajos anteriores $(8,9,10)$. Tanto en la Consulta Prenatal como en las salas de embarazo patólogico, de trabajo de parto, de puerperio, de aislamiento o de alto riesgo, están reunidas, sin la menor consideración, con las demás gestantes, recibiendo la mirada compasiva o escrutadora de cuantas personas las rodean en atención a su corta edad.

Este panorama nuestro, sin la menor duda, es el que se vive en la mayoría de los hospitales del mundo entero.

Creemos que el análisis ordenado que. acabamos de esbozar sea suficiente para iniciar una amplia discusión sobre la necesidad de constituir policlínicas especializadas para la atención ginecológica de las niñas y gíneco-obstétrica de las adolescentes $y$ estudiar su organización

\section{Experiencia nacional. Resultado de una encuesta}

\section{A. Programas universitarios}

En nuestro país ha existido de tiempo atrás una gran preocupación por el conocimiento y atención de los trastornos ginecológicos de las niñas y gínecoobstétricos de las adolescentes.

Una encuesta adelantada en 1972 por Pedraza y Camacho (14) en las 7 Facultades de Medicina que existían por aquel entonces, demostró que una facultad tenía un programa específico, con dedicación semestral de 3 horas para la teoría y 21 para la práctica. En 3, había 
una preocupación especial y dedicaban 36 horas a la parte teórica y 11 a la práctica, pero en un plano general, sin programas específicos. En 2 se estudiaba la posibilidad de adelantar cursillos de postgrado.

Nosotros, para tener una idea exacta de los Programas de Educación Médica en nuestro país, sobre el tópico que nos ocupa, enviamos una encuesta a 17 Facultades de Medicina de Colombia. Sólo 10 nos dieron respuesta, $58.82 \%$. Estas 10 facultades, por su ubicación geográfica, corresponden 3 a la región central de Colombia, 3 a la costa norte, 3 a la región occidental y una a la zona oriental.

Con los datos recibidos, haremos el análisis y los comentarios de lo que estamos haciendo en materia de enseñanza universitaria en este campo. Siete facultades tienen programada la enseñanza de Ginecología Infanto Juvenil: 2 como programa especial, 2 la inician en la Cátedra de Pediatría y la continúan en la de Ginecología, 3 la dictan en la Cátedra de Ginecología (Cuadro No. 2).

En una facultad, que no tiene programada la instrucción, se imparten conocimientos a los alumnos por iniciativa del profesorado, cada vez que se presenta la oportunidad. Dos facultades iniciarán programas de docencia en los próximos semestres, solamente una no ha decidido, programar esta enseñanza.

Cinco facultades tienen esta disciplina en los programas de internado y dos la incorporarán en breve tiempo. Dos dictan cursillos de entrenamiento en postgrado $y$ una ha enviado a especializarse al exterior a 5 de sus egresados, con el compromiso de vincularse luego a los programas docentes universitarios en este campo. En 8 facultades se ha observado especial interés por parte de profesores y alumnos para el estudio de esta especialidad.
Cuadro No. 2

METODOS DE ATENCION DE LA NIÑA Y DE LA ADOLESCENTE

\section{EXPERIENCIAS NACIONALES} COLOMBIANAS

Docencia en 10 Facultades de Medicina

\begin{tabular}{|lcc|}
\hline & Si & No \\
\hline Tienen programas de docencia & 7 & 3 \\
Con Cátedra especial & 2 & - \\
Incorporado a los de Pediatría & 2 & - \\
Incorporado a los de Ginecología & 3 & - \\
Se enseña sin programación & 1 & - \\
Pronto iniciarán programas & 2 & 1 \\
Tienen programa de internado & 5 & 5 \\
Iniciarán programa de internado & 3 & 2 \\
Cursillos de postgrado & 2 & 8 \\
Darán título de especialista & - & 10 \\
Hay interés en docentes & 8 & 2 \\
Hay interés en estudiantes & 8 & 2 \\
\hline
\end{tabular}

En nuestra encuesta no preguntamos las horas dedicadas por semestre a la enseñanza teórica ni a la práctica.

No hemos querido tomar el silencio de 7 facultades como respuesta negativa pues nos consta que en algunas de ellas se siguen programas similares a los expuestos anteriormente. Pensamos sí, que este mutismo revela la falta de un interés por los problemas ginecológicos de la niña y gíneco-obstétricos de la adolescente. Carecemos de una política nacional definida para el estudio de este apasionante capítulo de nuestra especialidad.

Sabemos que son muchas las causas que impiden adelantar a cabalidad estos programas de docencia en las Facultades de Medicina; entre otras, la falta de equipos profesionales especializados para tal fin, la brevedad del tiempo académico $y$ el exceso de estudiantes que deben 
atenderse, la falta de financiación, la carencia de planta básica, el reducido número de Sociedades o de Grupos de Estudio de Ginecología Infanto Juvenil que promuevan esta especialidad, más el concepto defendido por no pocos ginecólogos de que no debemos constituir subespecialidades dentro de nuestra especialidad. Con todo, consideramos que hemos avanzado en esta última década y vemos un panorama promisorio y firme.

\section{B. Atención Hospitalaria}

Enviamos una encuesta a 19 hospitales Universitarios o con programas de docencia e internado. Desafortunadamente, como en el caso de las Facultades de Medicina, no todas dieron respuesta: sólo 11 contestaron nuestro cuestionario.

Como norma en los hospitales del país las niñas hasta de 11 años se atienden en los servicios de pediatría; después de los once años van a los servicios generales del hospital, hecho que por demás, es prácticamente universal.

\section{Cuadro No. 3}

\section{METODOS DE ATENCION DE LA NIÑA $Y$ DE LA ADOLESCENTE}

\section{EXPERIENCIAS NACIONALES COLOMBIANAS}

Atención hospitalaria. Consulta externa.

\begin{tabular}{|lcr|}
\hline & Si & No \\
\hline De Ginecología Pediátrica & 6 & 5 \\
Atendida por equipo médico & 4 & 2 \\
Atendida por ginecólogo & 2 & - \\
De Ginecología para adolescentes & - & 11 \\
De prenatal para adolescentes & - & 11 \\
\multicolumn{1}{l}{ Horas promedio de consulta: 2} & & \\
\hline
\end{tabular}

\section{Consulta Externa}

De los 11 Hospitales que respondieron la encuesta, sólo 6 tienen establecida la CONSULTA EXTERNA DE GINECOLOGIA INFANTIL. En los 5 restantes, las niñas son atendidas en la Consulta General de Pediatría y tratadas allí o remitidas a la consulta general de Ginecología. Para finales del presente año un hospital universitario inaugura una planta física para Consulta Externa de Adolescentes.

Ninguno de los hospitales tiene establecida una consulta externa para atención ginecológica $u$ obstétrica de las mayores de once años: todas deben ir a los servicios de consulta ginecológica o de atención prenatal. En un hospital estas gestantes se atienden en la Consulta de Alto Riesgo.

De las 6 consultas externas de ginecología infantil, 4 son atendidas por un equipo médico especializado, constituido por Pediatra, Ginecólogo, Siquiatra Infantil, Endocrinólogo, Sicólogo, Trabajadora Social y Residente de Pediatría, a más de enfermeras y personal auxiliar entrenado. En dos hospitales, la consulta es atendida por un ginecólogo (cuadro No. 3).

Las horas de atención de esta Consulta Externa van de dos horas dos veces por semana hasta tres horas diarias, Io que nos da un promedio de dos horas diarias por hospital.

Los 11 hospitales mencionados nos informaron el número total de pacientes atendidas en el último año en sus Departamentos de Ginecología y Obstetricia; pero sólamente 8 rios dieron a conocer el número de menores de 11 años atendidas en Consulta Externa Ginecológica $\mathrm{y}$ el de menores de 16 años atendidas en prenatai. Ningún hospital nos informó el número de pacientes de 
11 a 16 años atendidas por Consulta ginecológica (cuadro No. 4).

El total de pacientes atendidas por consulta externa ginecológica en estos ocho hospitales fue de 21.961 y de ellas, el $9.9 \%$ correspondía al grupo de niñas menores de 11 años.

El total de pacientes atendidas por Consulta Prenatal fue de 47.195 y de ellas el $2.17^{\circ} \%$ fueron adolescentes menores de 16 años.

Estas cifras son muy significativas estadísticamente y nos hablan con elocuencia sobre la necesidad de organizar consulta externa especializada para la atención ginecológica de las niñas $y$ gíneco obstétrica de las adolescentes.

\section{Hospitalización}

A excepción de dos hospitales que tienen un servicio exclusivo para la atención ginecológica de las niñas menores de 11 años, los demás las remiten al Departamento de Pediatría o al Servicio de Endocrinología.
A las mayores de 11 años se les hospiza en el Servicio de Ginecología, de Endocrinología o de Maternidad y allí se les separa de las pacientes de mayor edad por biombos o canceles en tres centros hospitalarios; en los 8 restantes se les coloca indiscriminadamente con las demás gestantes sin tener consideración alguna con su edad. En un hospital todos los casos obstétricos se remiten al Servicio de Alto Riesgo (cuadro No. 5).

No conocemos el número de partos atendidos en los hospitales del país en menores de 16 años en los últimos doce meses. Por trabajos realizados por nosotros y por otros investigadores nacionales entre 1972 y $1978(8,9,11,13)$ sabemos que la incidencia de partos a esta edad ha sido del $2.3 \%$.

Observamos un aumento muy marcado año tras año en el número de adolescentes que acuden al Servicio de Prenatal y de Maternidad para tratamiento de abortos y sus complicaciones, de embarazo normal o patológico, para la atención del parto y el manejo del puerperio.

Conocemos también, por los estudios citados anteriormente, la baja utilización

Cuadro No. 4

METODOS DE ATENCION DE LA NIÑA $Y$ DE LA ADOLESCENTE

EXPERIENCIAS NACIONALES COLOMBIANAS

Pacientes atendidas en 8 hospitales por Consulta Externa

\begin{tabular}{|lcccc|}
\hline & Total & $\begin{array}{l}\text { Menores } \\
11 \text { años }\end{array}$ & $\begin{array}{l}\text { De 11 a } \\
16 \text { años }\end{array}$ & $\%$ \\
\cline { 2 - 5 } & 21.961 & 2.176 & $?$ & 9.9 \\
Ginecológica & 41.195 & - & 1.025 & 2.17 \\
Prenatal & & & & \\
\hline
\end{tabular}


Cuadro No. 5

\section{METODOS DE ATENCION DE LA NIÑA $Y$ DE LA ADOLESCENTE}

\section{EXPERIENCIAS NACIONALES COLOMBIANAS}

Atención Hospitalaria. Hospitalización

\begin{tabular}{|lcc|}
\hline & Sí & No \\
\cline { 2 - 3 } Sala ginecológica para menores 11 años & 2 & 9 \\
Se hospitalizan en Pediatría & 9 & - \\
Sala ginecológica para mayores 11 años & - & 11 \\
Se hospitalizan en Ginecología & 11 & - \\
Algunas van a Endocrinología & 3 & - \\
Sala maternidad para adolescentes & - & 11 \\
Se hospitalizan en maternidad general & 11 & - \\
Mayores 11 años se separan por cancel & 3 & 8 \\
Se hospitalizan en alto riesgo & 1 & 10 \\
\hline
\end{tabular}

de los diferentes métodos anticonceptivos por parte de eștas pacientes de tan corta edad que acuden a los servicios hospitalarios: el $96.1 \%$ no planificaban (13). Existe últimamente un buen número de adolescentes que aceptan y piden la anticoncepción después del aborto o del parto. Ello nos hace ver la necesidad de mejorar los programas de información y educación. Es éste un problema que se debe resolver con tino para no herir susceptibilidades. Requiere consejo, dirección y suministro de anticoncepción, teniendo en cuenta siempre factores sicológicos y biológicos, al mismo tiempo que, aspectos familiares, sociales y aún profesionales y éticos.

En nuestra encuesta nos abstuvimos de indagar datos sobre este tópico.
Cuadro No. 6

\section{METODOS DE ATENCION DE LA} NIÑA Y DE LA ADOLESCENTE

EXPERIENCIAS NACIONALES COLOMBIANAS

Niñas y adolescentes atendias en 8 hospitales

\begin{tabular}{llll}
\hline & $\begin{array}{c}\text { Consulta } \\
\text { Externas }\end{array}$ & $\begin{array}{c}\text { Hospita- } \\
\text { lizadas }\end{array}$ & $\%$ \\
\hline En Ginecología & 2.176 & 265 & 12.17 \\
En Obstetricia & 1.025 & 527 & 51.41 \\
\hline
\end{tabular}


EI $12.17 \%$ de las pacientes atendidas en Consulta Externa necesitaron hospitalización para tratamiento de problemas ginecológicos. Recordemos que estamos relacionando en nuestro estudio adolescentes únicamente hasta de 16 años.

Es alentador observar que el $51.41 \%$ de las adolescentes son atendidas en prenatal estuvieran luego hospitalizadas (cuadro No. 6); ello significa que no se ha perdido la campaña adelantada para que toda adolescente embarazada busque, no sólo la atención prenatal sino también el servicio interno hospitalario para la atención del parto o de las complicaciones que se le puedan presentar a cualquier edad de la gestación.

La Cirugía Ginecológica en diez hospitales la realiza un equipo constituido por un ginecólogo y un cirujano pediatra. En un hospital intervienen sólo los cirujanos pediatras, si bien en determinadas circunstancias se asesoran del ginecólogo (cuadro No. 7).

Cuadro No. 7

\section{METODOS DE ATENCION DE LA NIÑA Y DE LA ADOLESCENTE}

\section{EXPERIENCIAS NACIONALES COLOMBIANAS}

\section{Cirugía Ginecológica}

\begin{tabular}{lcc}
\hline & $\begin{array}{l}\text { Equipo ginecólogo } \\
\text { Cirujano Pediatra }\end{array}$ & $\begin{array}{l}\text { Cirujano } \\
\text { Pediatra }\end{array}$ \\
\cline { 2 - 3 } La practican & 10 & 1 \\
\hline
\end{tabular}

En los once hospitales se cuenta con la colaboración de todos los Servicios y Departamentos de la Institución. Consideramos que el Servicio Social es indispensable y básico como enlace entre la familia, el hospital y la paciente. EI instrumental y la dotación quirúrgica por lo general son suficientes y adecuados para el desarrollo de las mejores tecnicas. La colaboración que los Centros Oncológicos prestan a estos hospitales es oportuna y eficiente.

\section{Concepto sobre servicios especializados}

En el concepto de 8 hospitales, la consulta externa de Ginecología Infanto Juvenil y la Consulta prenatal deben ser especializadas $\mathrm{y}$ atendidas por personal idóneo y entrenado, con carácter de indispensable. Para 3 hospitales estos servicios son considerados como útiles, mas no indispensables (cuadro No. 8).

$$
\text { Cuadro No. } 8
$$

METODOS DE ATENCION DE LA NIÑA $Y$ DE LA ADOLESCENTE

EXPERIENCIAS NACIONALES COLOMBIANAS

Concepto sobre atención especializada

\begin{tabular}{|lccc|}
\hline & Indispensable & Util & Innecesaria \\
\cline { 2 - 5 } Consulta Ginecológica & 8 & 3 & - \\
Consulta Prenatal & 8 & 3 & - \\
Servicio Ginecología & 8 & 3 & - \\
Servicio Maternidad & 8 & 3 & - \\
\hline
\end{tabular}

Un concepto similar existe en igual número de hospitales en relación con los servicios internos para la atención médica o quirúrgica de estas pacientes. Ningún hospital ha considerado estos servicios como innecesarios. Estos conceptos quieren decir que hay un consenso general sobre la necesidad de organizar dentro de los Departamentos de Pediatría y de Ginecología los Servicios de Consulta Externa especializada como también las Salas para la atención exclusiva de estas pacientes. 
Estos mismos hospitales conceptúan que para la atención de la consulta externa y de los servicios de hospitalización se requieren equipos médicos entrenados, constituidos por Pediatra, Ginecólogo, Siquiatra Infantil, Sicólogo, Endocrinólogo, a más de enfermeras y personal auxiliar adiestrado.

Nos faltan políticas de orientación para la organización de estos servicios y fondos económicos suficientes para su funcionamiento. El personal médico y auxiliar está en un período franco de adiestramiento y capacitación.

Para 9 hospitales el adiestramiento y los cursillos de información a todo nivel son indispensables y para 2 , son de utilidad (cuadro No. 9).

Cuadro No. 9

\section{METOdOS DE ATENCION DE LA NIÑA $Y$ DE LA ADOLESCENTE}

EXPERIENCIAS NACIONALES COLOMBIANAS

Concepto sobre adiestramiento y cursillos

\begin{tabular}{|c|c|c|c|}
\hline & Indispensable & Util & Innecesario \\
\hline Para Médicos & 9 & 2 & - \\
\hline Personal auxiliar & 9 & 2 & - \\
\hline \multicolumn{4}{|c|}{ TODOS BRINDAN OPORTUNIDAD } \\
\hline
\end{tabular}

Los cursillos exigen erogación económica; desafortunadamente las limitaciones presupuestales y aún locativas, como la falta de personal adiestrado, dificultan y aún impiden el desarrollo de estos programas, tanto de educación como asistenciales, considerados indispensables por la razón de los hechos.

Cabe destacar el trabajo que de años atrás vienen desarrollando al respecto la Academia Nacional de Medicina, la SO-
CIEDAD COLOMBIANA DE GINECOLOGIA JUVENIL Y PEDIATRICA Y sus filiales por el logro de la implantación de programas de docencia y de atención ginecológica a niñas y adolescentes en todas las facultades de medicina $y$ en todos los hospitales del país.

\section{BASES FOR SPECIALIZED GYNECOLOGICAL CARE TO GIRLS AND ADOLESCENTS}

\section{Summary}

The bases for a specialized gynecological care to girls and adolescents are stated. A study is made on the bases which permit the organization of these specialized services: Education to the community, medical and paramedical training and instruction and offering of both external consultation and hospitalization services within the Pediatrics and GinecoObstetrics Department of the Aid Institutions. Finally, the results of a survey made among 17 Medicine Faculties and 20 hospitals in Colombia regarding teaching programs, hospital attention and projects to be executed in the near future in relation with the subject herein discussed, are shown.

\section{Resumen}

Se exponen los fundamentos para una atención ginecológica especializada a niñas y adolescentes. Se estudian las bases que hacen posible la organización de estos servicios especializados: educación de la comunidad, capacitación y adiestramiento médico y paramédico y apertura de servicios de Consulta Externa y de hospitalización dentro de los Departamentos de Pediatría y de Gíneco-Obste- 
tricia de las Instituciones de Asistencia. Se presenta, por último, el resultado de una encuesta adelantada en 17 Facultades de Medicina y en 20 hospitales de

\section{Bibiografía}

1. AMAYA L.H. y GARCIA, C.C. Vaginitis en niñas. Consideraciones etiológicas en Bogotá. Rev. Colom. Obst. Gin. 24: 273, 1973.

2. CARDONA, A.F. Adolescencia. Rev. Colomb. Obst. Gin. 28: 53, 1977.

3 Compte Rendu d' activité de la FIGIJ au cours de I' exercise 1972-1973. Pág. 2 y 3.

4. Compte Rendu d' activité de la FIGIJ au cours de l'exercice 1977-1979. Boulletin No. 5.

5. DUARTE-CONTRERAS, A. Fisiología de las hormonas sexuales en la infancia Rev. Colomb. Obst. Gin. 22: 385, 1971.

6. DUARTE-CONTRERAS, A. Estudio bacteriológico de secreción vaginal en 200 niñas asintomáticas, menores de 12 años. Rev. Colomb. Obst. Gin. 26: 381, 1975.

7. DUARTE-CONTRERAS, A. Disgerminoma en niñas y adolescentes. Rev. ColomObst. Gin. 30: 227, 1979.

8. DUARTE-CONTRERAS, A. Embarazo en adolescentes de 11 a 15 años. Rev. Colomb. Obst. Gin. 24: 259, 1973.

9. DUARTE-CONTRERAS, A. ¿EI emba razo en adolescentes es siempre de alto riesgo? Rev. Colomb. Obst. Gin. 26: 385, 1975.

10. DUARTE-CONTRERAS, A. Embarazo y prostitución en adolescentes. Rev. Colomb. Obst. Gin. 30: 1, 1979.
Colombia sobre programas de docencia, atención hospitalaria y proyectos de próxima realización en torno al tema que nos ocupa.

11. GOMEZ, P.J.A. Obstetricia en adolescentes. Rev. Colomb. Obst. Gin. 23: 353, 1972.

12. JUBIZ, A. y COLS. Motivos de consultas más frecuentes en $\mathbf{6 2 0}$ adolescentes. Rev. Colomb. Obst. Gin. 23: 399, 1972.

13. LOPEZ, E.G. y COLS. La embarazada adolescente. CCRP. Avances en Obstetricia y Ginecología. Memorias II Curso internacional. Vol. 14: 109, 1979, Bogotá.

14. PEDRAZA, H. y CAMACHO, G.J. Encuesta sobre la introducción del estudio del adolescente dentro de los programas de educación médica en siete universidades colombianas. En "Temas Médicos", órgano oficial de la Academia Nacional de Medicina de Colombia. Edit. Stella. Tomo VII: 281, 1973.

15. PEREZ, G.E., PARADA, R.C., DUARTE CONTRERAS, A. y LUZARDO, M.L.F., Tumores de ovario. Rev. Colomb. Obst. Gin. 26: 369, 1975.

\section{NOTA:}

Nuestro agradecimiento a las Facultades de Medicina y a los Hospitales que respondieron nuestra encuesta haciendo así posible la elaboración del presente trabajo y su presentación como Ponencia Oficial ante el VI Simposio Mundial de Ginecología de la Niña y la Adolescente (Punta del Este, Uruguay, diciembre 4 de 1981).

\section{ALBERTO DUARTE CONTRERAS}

Cúcuta, octubre 3 de 1981

Apartado aéreo 849 